ESAIM: M2AN

M2AN, Vol. 41, No 1, 2007, pp. 77-93

DOI: $10.1051 / \mathrm{m} 2 \mathrm{an}: 2007008$
ESAIM: Mathematical Modelling and Numerical Analysis

www.edpsciences.org/m2an

\title{
CONVERGENCE OF THE TIME-DISCRETIZED MONOTONIC SCHEMES*
}

\author{
JULIEN SALOMON ${ }^{1,2}$
}

\begin{abstract}
Many numerical simulations in (bilinear) quantum control use the monotonically convergent Krotov algorithms (introduced by Tannor et al. [Time Dependent Quantum Molecular Dynamics (1992) 347-360]), Zhu and Rabitz [J. Chem. Phys. (1998) 385-391] or their unified form described in Maday and Turinici [J. Chem. Phys. (2003) 8191-8196]. In Maday et al. [Num. Math. (2006) 323-338], a time discretization which preserves the property of monotonicity has been presented. This paper introduces a proof of the convergence of these schemes and some results regarding their rate of convergence.
\end{abstract}

Mathematics Subject Classification. 49J20, 68W40.

Received: January 2, 2006. Revised: November 3, 2006.

\section{INTRODUCTION}

Quantum control has recently been subjected to significant developments through encouraging experimental results [7, 15]. At computational level [4], the introduction of monotonic Krotov algorithms (introduced by Tannor et al. in [18]), Zhu and Rabitz [20] or the unified form proposed by Maday and Turinici in [10] has enabled us to design efficient methods being implemented to obtain laser fields that control the molecular dynamics [13]. From a mathematical point of view, it can be proved that these procedures monotonically increase the values of a given criterium. Yet, few results are available about the convergence of the control sequences obtained by these schemes. In [14], a first necessary condition for convergence has been obtained in case of large penalization of the $L^{2}$-norm of the control. A functional analysis of the schemes has been displayed in [6] in the abstract framework of semi-group theory. To complete these works, a first analysis of a time-discretized version of the monotonic algorithms is presented here.

Let us briefly introduce the model and the corresponding optimal control framework used in this paper. Consider a quantum system described by its wavefunction $\psi=\psi(x, t)$. The relevant spatial coordinates, denoted by $x$, belong to a general space $\Omega \subset \mathbb{R}^{3 p}$, where $p$ is the number of particles considered (the symbol $x$ will often be omitted in the following developments in order to make it simple). Absorbing boundary conditions [17] can be used to treat numerically the case of unbounded domains. We assume homogeneous Dirichlet

Keywords and phrases. Quantum control, monotonic schemes, optimal control, Łojasiewicz inequality.

* The author acknowledges financial support from the Action Concertée Incitative "Simulation Moléculaire" of the MENRT (France).

1 Université Pierre et Marie Curie, Paris 6, Laboratoire Jacques-Louis Lions, 175 rue du Chevaleret 75013 Paris, France.

2 Université Paris-Dauphine, Paris 9, CEREMADE, Place du Maréchal Lattre de Tassigny, 75775 Paris Cedex 16, France.

Julien.Salomon@dauphine.fr

(c) EDP Sciences, SMAI 2007 
boundary conditions. The dynamics of this system is characterized by its internal Hamiltonian:

$$
H=H_{0}+V .
$$

In this equation $H_{0}$ is the kinetic part:

$$
H_{0}=-\frac{1}{2} \sum_{n=1}^{p} \frac{1}{m_{n}} \Delta_{r_{n}}
$$

where $m_{n}$ is the mass of the particle $n$ and $\Delta_{r_{n}}$ the Laplace operator with respect to its coordinates. The operator $V=V(x)$ is the potential part. In case the domain is bounded and $V$ is smooth, the spectrum of $H$ is discrete, see e.g. [2]. In corresponding numerical simulations, spectral decompositions can be used to discretize the wavefunction.

A way to control such a system is to light it with a laser pulse modelled by a scalar electric field $\varepsilon(t)$. The contribution of this laser field is taken into account by introducing a perturbative term in the Hamiltonian which then reads $H-\mu \varepsilon$. Like $V$, the dipolar momentum $\mu$ is a function of $x$. In many models, the value $\mu(x)=+\infty$ is only reached for $|x|=+\infty$. Neglecting the interactions with far-off particles, we suppose that the operator $\mu$ is bounded on $L^{2}(\Omega)$. The evolution of $\psi$ is then governed by the Schrödinger equation (we are making use of atomic units, i.e. $\hbar=1)$ :

$$
\begin{aligned}
& i \frac{\partial}{\partial t} \psi=H \psi-\mu \varepsilon \psi \\
& \psi(t=0)=\psi_{\text {init }},
\end{aligned}
$$

where $\psi_{\text {init }}$ is the initial condition for $\psi$, subjected to the constraint: $\left\|\psi_{\text {init }}\right\|_{L^{2}(\Omega)}=1$. In numerical simulations, the ground state, i.e. a unitary eigenvector of $H$ associated to the lowest eigenvalue, is generally taken as the initial state. The Hamiltonian being real, the evolution through (1) preserves the $L^{2}$-norm of $\psi$ so that

$$
\|\psi(t)\|_{L^{2}(\Omega)}=1 .
$$

Remark 0.1. The analysis presented here after will be carried out in a general setting and prevails for all space discretizations preserving property (2).

In order to design relevant fields of control, the optimal control framework usually introduces a cost functional to assess their quality. A general example of such functional is given by:

$$
J(\varepsilon)=\langle\psi(T)|O| \psi(T)\rangle-\alpha \int_{0}^{T} \varepsilon^{2}(t) \mathrm{d} t,
$$

where $\alpha$ and $T$ are two positive real numbers and $O$ is a positive symmetric operator, also called observable, that encodes the target: the larger the value $\langle\psi(T)|O| \psi(T)\rangle$ is, the better the control objectives are met (here and in what follows, we note $\langle f|A| g\rangle=\int_{\Omega} \overline{f(x)} A g(x) \mathrm{d} x$, where $f$ and $g$ are complex valued functions and $A$ a given operator). In this paper, we assume that $O$ is bounded on $L^{2}(\Omega)$. This assumption is true in many physical models. However, the analysis presented here after is relevant if $O$ is only bounded on sub-domains containing the system. The Euler-Lagrange equations corresponding to $J$ can be written by means of the introduction of a Lagrange multiplier $\chi$, called adjoint state in what follows. The system obtained by this way is [20]:

$$
\begin{aligned}
& \left\{\begin{array}{l}
i \frac{\partial}{\partial t} \psi=(H-\mu \varepsilon) \psi \\
\psi(t=0)=\psi_{\text {init }}
\end{array}\right. \\
& \left\{\begin{array}{l}
i \frac{\partial}{\partial t} \chi=(H-\mu \varepsilon) \chi \\
\chi(t=T)=O \psi(T)
\end{array}\right. \\
& \alpha \varepsilon(t)=-\operatorname{Im}\langle\chi(t)|\mu| \psi(t)\rangle .
\end{aligned}
$$


An efficient tool to solve these equations is provided by the monotonic schemes. A general formulation of these algorithms is given by the following system (see [10]), which is defined recursively:

$$
\begin{aligned}
& \left\{\begin{array}{l}
i \frac{\partial}{\partial t} \psi^{k}=\left(H-\mu \varepsilon^{k}\right) \psi^{k} \\
\psi^{k}(t=0)=\psi_{\text {init }}
\end{array}\right. \\
& \varepsilon^{k}(t)=(1-\delta) \widetilde{\varepsilon}^{k-1}(t)-\frac{\delta}{\alpha} \operatorname{Im}\left\langle\chi^{k-1}(t)|\mu| \psi^{k}(t)\right\rangle \\
& \left\{\begin{array}{l}
i \frac{\partial}{\partial t} \chi^{k}=\left(H-\mu \widetilde{\varepsilon}^{k}\right) \chi^{k} \\
\chi^{k}(t=T)=O \psi^{k}(T)
\end{array}\right. \\
& \widetilde{\varepsilon}^{k}(t)=(1-\eta) \varepsilon^{k}(t)-\frac{\eta}{\alpha} \operatorname{Im}\left\langle\chi^{k}(t)|\mu| \psi^{k}(t)\right\rangle,
\end{aligned}
$$

where $\delta, \eta \in[0,2]$, with $(\delta, \eta) \neq(0,0)$. A significant property of these schemes is that the cost functional values monotonically increase during the iterations since:

$$
\begin{aligned}
J\left(\varepsilon^{k+1}\right)-J\left(\varepsilon^{k}\right)= & \left\langle\psi^{k+1}(T)-\psi^{k}(T)|O| \psi^{k+1}(T)-\psi^{k}(T)\right\rangle \\
& +\left(\frac{2}{\delta}-1\right) \int_{0}^{T} \alpha\left(\varepsilon^{k+1}(t)-\tilde{\varepsilon}^{k}(t)\right)^{2} \mathrm{~d} t+\left(\frac{2}{\eta}-1\right) \int_{0}^{T} \alpha\left(\tilde{\varepsilon}^{k}(t)-\varepsilon^{k}(t)\right)^{2} \mathrm{~d} t \geq 0 .
\end{aligned}
$$

Implicit and explicit relevant time discretizations of these schemes have been designed and tested in [11,12]. Their main property is to maintain the monotonic character at the discrete level. This paper aims at proving the convergence of these algorithms.

The paper is structured as follows: the time discretizations of the Schrödinger equation (1) and the cost functional defined in (3) will be presented in Section 1. In Section 2, we will study the variations in the discrete cost functional with respect to $\varepsilon$. In Section 3, we will recall the main features of the implicit time discretized monotonic schemes. Section 4 is dedicated to proving the convergence of these schemes. Finally, we will give some estimates of the rate of convergence in Section 5. Sufficient condition of convergence of the explicit scheme is provided in the appendix.

\section{Discrete optimal Control Setting}

For any given integer $N$, let us introduce the discretization parameter $\Delta T$ defined by $N \Delta T=T$ and $\varepsilon_{j}$, $\tilde{\varepsilon}_{j}, \psi_{j}, \chi_{j}$ that stand respectively for approximations of $\varepsilon(j \Delta T), \tilde{\varepsilon}(j \Delta T), \psi(j \Delta T), \chi(j \Delta T)$. We denote in the following $\varepsilon=\left(\varepsilon_{j}\right)_{0 \leq j \leq N-1}, \tilde{\varepsilon}=\left(\tilde{\varepsilon}_{j}\right)_{0 \leq j \leq N-1}, \psi=\left(\psi_{j}\right)_{0 \leq j \leq N}$ and $\chi=\left(\chi_{j}\right)_{0 \leq j \leq N}$.

To solve (1) numerically with enough accuracy, a propagation method based on the Strang's second-order split-operator technique $[16]$ is generally used (see e.g. $[1,19,20]$ ). This method also simplifies algebraic manipulations as it will appear later in this study (see Eq. (21)).

In this part, for any prescribed sequences $\varepsilon, \tilde{\varepsilon}$, the approximations of the state $\psi$ and the adjoint state $\chi$ are thus defined by the semi-discretized propagation equations:

$$
\begin{aligned}
& \left\{\begin{array}{l}
\psi_{j+1}^{\varepsilon}=\mathrm{e}^{\frac{H_{0} \Delta T}{2 i}} \mathrm{e}^{\frac{V-\mu \varepsilon_{j}}{i} \Delta T} \mathrm{e}^{\frac{H_{0} \Delta T}{2 i}} \psi_{j}^{\varepsilon} \\
\psi_{0}^{\varepsilon}=\psi_{\text {init }},
\end{array}\right. \\
& \left\{\begin{array}{l}
\chi_{j}^{\tilde{\varepsilon}}=\mathrm{e}^{-\frac{H_{0} \Delta T}{2 i}} \mathrm{e}^{\frac{-V+\mu \tilde{\varepsilon}_{j}}{i} \Delta T} \mathrm{e}^{-\frac{H_{0} \Delta T}{2 i}} \chi_{j+1}^{\tilde{\varepsilon}} \\
\chi_{N}^{\tilde{\varepsilon}}=O \psi_{N}^{\varepsilon} .
\end{array}\right.
\end{aligned}
$$


Note that actually $\chi^{\tilde{\varepsilon}}$ also depends on $\varepsilon$. We omit this dependence for purpose of simplicity. We refer to [12] for more details about full discretization. This discretization of (4) and (5) preserves the $L^{2}$-norm of the propagated vectors, which means:

$$
\begin{array}{r}
\forall j=0 \ldots N-1, \quad\left\|\psi_{j}^{\varepsilon}\right\|_{L^{2}(\Omega)}=\left\|\psi_{\text {init }}\right\|_{L^{2}(\Omega)}=1, \\
\left\|\chi_{j}^{\tilde{\varepsilon}}\right\|_{L^{2}(\Omega)}=\left\|\chi_{N}^{\tilde{\varepsilon}}\right\|_{L^{2}(\Omega)} \leq\|O\|_{*},
\end{array}
$$

where $\|O\|_{*}$ denotes the norm of the operator $O$ over $L^{2}(\Omega)$. We consider the following time discretization of the cost functional defined in (3):

$$
J_{\Delta T}(\varepsilon)=\left\langle\psi_{N}^{\varepsilon}|O| \psi_{N}^{\varepsilon}\right\rangle-\alpha \Delta T \sum_{j=0}^{N-1}\left|\varepsilon_{j}\right|^{2} .
$$

Let us also introduce norms on $\mathbb{R}^{N}$ corresponding to the time discretization:

$$
\|\varepsilon\|_{1}=\Delta T \sum_{j=0}^{N-1}\left|\varepsilon_{j}\right|,\|\varepsilon\|_{2}=\left(\Delta T \sum_{j=0}^{N-1}\left|\varepsilon_{j}\right|^{2}\right)^{\frac{1}{2}} .
$$

Note that the inner product associated with the norm $\|\cdot\|_{2}$ is defined by:

$$
\varepsilon . \varepsilon^{\prime}=\Delta T \sum_{j=0}^{N-1} \varepsilon_{j} \varepsilon_{j}^{\prime} .
$$

\section{VARIATIONS IN $J_{\Delta T}$}

In this section, we investigate some variational properties of $J_{\Delta T}$.

\subsection{Gradient of $J_{\Delta T}$}

Given a field $\varepsilon$, the dependence of $\psi_{N}^{\varepsilon}$ with respect to the field can be explicitly stated with the formula:

$$
\psi_{N}^{\varepsilon}=\prod_{j=0}^{N-1}\left(\mathrm{e}^{\frac{H_{0} \Delta T}{2 i}} \mathrm{e}^{-i\left(V-\mu \varepsilon_{j}\right) \Delta T} \mathrm{e}^{\frac{H_{0} \Delta T}{2 i}}\right) \psi_{\text {init }} .
$$

The computation of the gradient of $J_{\Delta T}$ is simplified by the introduction of the adjoint state $\chi^{\varepsilon}$. Indeed, note first that the partial derivative of $\left\langle\psi_{N}^{\varepsilon}|O| \psi_{N}^{\varepsilon}\right\rangle$ with respect to $\varepsilon_{j_{0}}$ reads:

$$
\frac{\partial \psi_{N}^{\varepsilon}}{\partial \varepsilon_{j_{0}}}=\Delta T \prod_{j=j_{0}+1}^{N-1}\left(\mathrm{e}^{\frac{H_{0} \Delta T}{2 i}} \mathrm{e}^{\frac{V-\mu \varepsilon_{j}}{i} \Delta T} \mathrm{e}^{\frac{H_{0} \Delta T}{2 i}}\right) \times\left(\mathrm{e}^{\frac{H_{0} \Delta T}{2 i}} \mathrm{e}^{\frac{V-\mu \varepsilon_{j_{0}}}{i} \Delta T} i \mu \mathrm{e}^{\frac{H_{0} \Delta T}{2 i}}\right) \times \prod_{j=0}^{j_{0}-1}\left(\mathrm{e}^{\frac{H_{0} \Delta T}{2 i}} \mathrm{e}^{\frac{V-\mu \varepsilon_{j}}{i} \Delta T} \mathrm{e}^{\frac{H_{0} \Delta T}{2 i}}\right) \psi_{\text {init }} .
$$

Consequently, the following equations hold true:

$$
\frac{\partial\left\langle\psi_{N}^{\varepsilon}|O| \psi_{N}^{\varepsilon}\right\rangle}{\partial \varepsilon_{j_{0}}}=2 \operatorname{Re}\left\langle\frac{\partial \psi_{N}^{\varepsilon}}{\partial \varepsilon_{j_{0}}}|O| \psi_{N}^{\varepsilon}\right\rangle=-2 \Delta T \operatorname{Im}\left\langle\widetilde{\chi}_{j_{0}}^{\varepsilon}|\mu| \breve{\psi}_{j_{0}}^{\varepsilon}\right\rangle
$$

where:

$$
\tilde{\chi}_{j}^{\varepsilon}=\mathrm{e}^{\frac{H_{0} \Delta T}{2 i}} \chi_{j}^{\varepsilon}, \quad \breve{\psi}_{j}^{\varepsilon}=\mathrm{e}^{\frac{H_{0} \Delta T}{2 i}} \psi_{j}^{\varepsilon} .
$$


The gradient of $J_{\Delta T}$ is then given by:

$$
\nabla J_{\Delta T}(\varepsilon) . \delta \varepsilon=-2 \Delta T \sum_{j=0}^{N-1}\left(\operatorname{Im}\left\langle\widetilde{\chi}_{j}^{\varepsilon}|\mu| \breve{\psi}_{j}^{\varepsilon}\right\rangle+\alpha \varepsilon_{j}\right) \delta \varepsilon_{j}
$$

The previous computation allows us to define set $C$ of the critical points of $J_{\Delta T}$ :

$$
C=\left\{\varepsilon / \forall j=0 \ldots N-1, \operatorname{Im}\left\langle\widetilde{\chi}_{j}^{\varepsilon}|\mu| \breve{\psi}_{j}^{\varepsilon}\right\rangle+\alpha \varepsilon_{j}=0\right\}
$$

\subsection{About the Hessian of $J_{\Delta T}$}

For some reasons that will be developed in Section 4.1, it can be useful to find conditions to make sure that the Hessian matrix $H_{J_{\Delta T}}(\varepsilon)$ of $J_{\Delta T}$ is invertible.

Denoting by $\left(\nabla J_{\Delta T}(\varepsilon)\right)_{\ell}$ the $\ell$-th component of $\nabla J_{\Delta T}(\varepsilon)$, the following equation prevails:

$$
\frac{\partial}{\partial \varepsilon_{m}}\left(\nabla J_{\Delta T}(\varepsilon)\right)_{\ell}=-2\left(\operatorname{Im}\left\langle\frac{\partial}{\partial \varepsilon_{m}} \tilde{\chi}_{\ell}^{\varepsilon}|\mu| \breve{\psi}_{\ell}^{\varepsilon}\right\rangle+\operatorname{Im}\left\langle\tilde{\chi}_{\ell}^{\varepsilon}|\mu| \frac{\partial}{\partial \varepsilon_{m}} \breve{\psi}_{\ell}^{\varepsilon}\right\rangle+\alpha \delta_{\ell, m}\right)
$$

where $\delta_{\ell, m}$ is the Kronecker's symbol. Repeating the analysis carried out in the previous section, we find out that $H_{J_{\Delta T}}(\varepsilon)$ reads:

$$
H_{J_{\Delta T}}(\varepsilon)=-2(S(\varepsilon)+\alpha I d)
$$

where $I d$ is the identity matrix and $S(\varepsilon)=\left(s_{\ell, m}\right)_{\substack{\ell=0 . . N-1 \\ m=0 . . N-1}}$ has the following property (see $(9)$ and $\left.(10)\right)$ :

$$
\left|s_{\ell, m}\right| \leq 2 \Delta T\|\mu\|_{*}^{2}\|O\|_{*},
$$

where $\|\mu\|_{*}$ is the norm of the operator $\mu$ on $L^{2}(\Omega)$. This estimate enables us to claim the following result.

Lemma 2.1. Let be $\varepsilon \in \mathbb{R}^{N}$. Suppose that $\alpha>2\|\mu\|_{*}^{2}\|O\|_{*} T$. Then $H_{J_{\Delta T}}(\varepsilon)$ is invertible.

Proof. Let us denote by $h_{\ell, m}$ the coefficients of $H_{J_{\Delta T}}(\varepsilon)$. According to (13), we obtain, for any $\ell=0 \ldots N-1$ :

$$
\begin{aligned}
\left|h_{\ell, \ell}\right| & \geq 2\left(\alpha-2 \Delta T\|\mu\|_{*}^{2}\|O\|_{*}\right) \\
& >4\|\mu\|_{*}^{2}\|O\|_{*}(T-\Delta T) \\
& \geq \sum_{m=0, m \neq \ell}^{N-1}\left|h_{m, \ell}\right| .
\end{aligned}
$$

Thus, $H_{J_{\Delta T}}(\varepsilon)$ is a dominant diagonal matrix and the result comes as a consequence.

\subsection{Variations in $\psi^{\varepsilon}$ and $\chi^{\varepsilon}$}

The variations in the state $\psi^{\varepsilon}$ and adjoint state $\chi^{\varepsilon}$ with respect to $\varepsilon$ can also be estimated by means of direct computations. Given two discrete fields $\varepsilon^{\prime}$ and $\varepsilon$, the difference $\psi_{j+1}^{\varepsilon^{\prime}}-\psi_{j+1}^{\varepsilon}$ reads:

$$
\psi_{j+1}^{\varepsilon^{\prime}}-\psi_{j+1}^{\varepsilon}=\mathrm{e}^{\frac{H_{0} \Delta T}{2 i}}\left(\mathrm{e}^{\frac{V-\mu \varepsilon_{j}^{\prime}}{i} \Delta T}-\mathrm{e}^{\frac{V-\mu \varepsilon_{j}}{i} \Delta T}\right) \mathrm{e}^{\frac{H_{0} \Delta T}{2 i}} \psi_{j}^{\varepsilon}+\mathrm{e}^{\frac{H_{0} \Delta T}{2 i}} \mathrm{e}^{\frac{V-\mu \varepsilon_{j}^{\prime}}{i} \Delta T} \mathrm{e}^{\frac{H_{0} \Delta T}{2 i}}\left(\psi_{j}^{\varepsilon^{\prime}}-\psi_{j}^{\varepsilon}\right) .
$$

The mean value inequality then yields:

$$
\left\|\psi_{j+1}^{\varepsilon^{\prime}}-\psi_{j+1}^{\varepsilon}\right\|_{L^{2}(\Omega)} \leq \Delta T\|\mu\|_{*}\left|\varepsilon_{j}^{\prime}-\varepsilon_{j}\right|+\left\|\psi_{j}^{\varepsilon^{\prime}}-\psi_{j}^{\varepsilon}\right\|_{L^{2}(\Omega)} .
$$


Hence:

$$
\left\|\psi_{j}^{\varepsilon^{\prime}}-\psi_{j}^{\varepsilon}\right\|_{L^{2}(\Omega)} \leq \Delta T\|\mu\|_{*} \sum_{l=0}^{j-1}\left|\varepsilon_{l}^{\prime}-\varepsilon_{l}\right| \leq\|\mu\|_{*}\left\|\varepsilon^{\prime}-\varepsilon\right\|_{1} .
$$

According to a similar argument:

$$
\left\|\chi_{j}^{\varepsilon^{\prime}}-\chi_{j}^{\varepsilon}\right\|_{L^{2}(\Omega)} \leq \Delta T\|\mu\|_{*}\left|\varepsilon_{j}^{\prime}-\varepsilon_{j}\right|+\left\|\chi_{j+1}^{\varepsilon^{\prime}}-\chi_{j+1}^{\varepsilon}\right\|_{L^{2}(\Omega)},
$$

and consequently:

$$
\begin{aligned}
\left\|\chi_{j}^{\varepsilon^{\prime}}-\chi_{j}^{\varepsilon}\right\|_{L^{2}(\Omega)} & \leq \Delta T\|\mu\|_{*} \sum_{l=j}^{N-1}\left|\varepsilon_{l}^{\prime}-\varepsilon_{l}\right|+\|O\|_{*}\left\|\psi_{N}^{\varepsilon^{\prime}}-\psi_{N}^{\varepsilon}\right\|_{L^{2}(\Omega)} \\
& \leq\|\mu\|_{*}\left\|\varepsilon^{\prime}-\varepsilon\right\|_{1}+\|O\|_{*}\left\|\psi_{N}^{\varepsilon^{\prime}}-\psi_{N}^{\varepsilon}\right\|_{L^{2}(\Omega)} \\
& \leq\|\mu\|_{*}\left(1+\|O\|_{*}\right)\left\|\varepsilon^{\prime}-\varepsilon\right\|_{1} .
\end{aligned}
$$

Remark 2.2. For algorithmic reasons, the adjoint state may be propagated with a field $\tilde{\varepsilon}$ that does not coincide with $\varepsilon$ (see (6)), the field bringing about the final condition $\chi_{N}^{\tilde{\varepsilon}}=O \psi_{N}^{\varepsilon}$. However, the previous estimate still holds insofar as we have:

$$
\begin{aligned}
\left\|\chi_{j}^{\tilde{\varepsilon}^{\prime}}-\chi_{j}^{\tilde{\varepsilon}}\right\|_{L^{2}(\Omega)} & \leq\|\mu\|_{*}\left\|\tilde{\varepsilon}^{\prime}-\tilde{\varepsilon}\right\|_{1}+\|O\|_{*}\left\|\psi_{N}^{\varepsilon^{\prime}}-\psi_{N}^{\varepsilon}\right\|_{L^{2}(\Omega)} \\
& \leq\|\mu\|_{*}\left(\left\|\tilde{\varepsilon}^{\prime}-\tilde{\varepsilon}\right\|_{1}+\|O\|_{*}\left\|\varepsilon^{\prime}-\varepsilon\right\|_{1}\right) .
\end{aligned}
$$

\section{IMPLiCit DisCRETE MONOTONIC SCHEMES}

This section aims at recalling the definition of the implicit discrete monotonic schemes and presenting some of their properties.

\subsection{Definition of the schemes}

Consider two real numbers $\delta, \eta \in\left[0,2\left[\right.\right.$ and the operator $\mu^{*}(h)$ defined by:

$$
\mu^{*}(h)=\frac{\mathrm{e}^{-i \mu h \Delta T}-I d}{-i h \Delta T}
$$

This function is an approximation of $\mu$ inasmuch as:

$$
\left\|\mu^{*}(h)-\mu\right\|_{*} \leq \Delta T\|\mu\|_{*}^{2}|h|
$$

which can be obtained by the mean value inequality, since $\left\|\frac{\mathrm{d} \mu^{*}(h)}{\mathrm{d} h}\right\|_{*} \leq \Delta T\|\mu\|_{*}^{2}$. 
Given initial control fields $\varepsilon^{0}, \tilde{\varepsilon}^{0}$ and their associated state $\psi^{0}$ and adjoint state $\chi^{0}$, suppose that for some $k \geq 1, \psi^{k-1}, \chi^{k-1}, \varepsilon^{k-1}, \tilde{\varepsilon}^{k-1}$ are known. The computation of $\psi^{k}, \chi^{k}, \varepsilon^{k}, \tilde{\varepsilon}^{k}$ is achieved as follows:

$$
\begin{aligned}
& \left\{\begin{aligned}
\psi_{j+1}^{k} & =\mathrm{e}^{\frac{H_{0} \Delta T}{2 i}} \mathrm{e}^{\frac{V-\mu \varepsilon_{j}^{k}}{i} \Delta T} \mathrm{e}^{\frac{H_{0} \Delta T}{2 i}} \psi_{j}^{k} \\
\varepsilon_{j}^{k} & =(1-\delta) \tilde{\varepsilon}_{j}^{k-1}-\frac{\delta}{\alpha} \operatorname{Im}\left\langle\widetilde{\chi}_{j}^{k-1}\left|\mu^{*}\left(\tilde{\varepsilon}_{j}^{k-1}-\varepsilon_{j}^{k}\right)\right| \breve{\psi}_{j}^{k}\right\rangle \\
\psi_{0}^{k} & =\psi_{\text {init }},
\end{aligned}\right. \\
& \left\{\begin{array}{l}
\chi_{j}^{k}=\mathrm{e}^{-\frac{H_{0} \Delta T}{2 i}} \mathrm{e}^{\frac{-V+\mu \tilde{\varepsilon}_{j}^{k}}{i} \Delta T} \mathrm{e}^{-\frac{H_{0} \Delta T}{2 i}} \chi_{j+1}^{k} \\
\tilde{\varepsilon}_{j}^{k}=(1-\eta) \varepsilon_{j}^{k}-\frac{\eta}{\alpha} \operatorname{Im}\left\langle\breve{\chi}_{j}^{k}\left|\mu^{*}\left(\varepsilon_{j}^{k}-\tilde{\varepsilon}_{j}^{k}\right)\right| \widetilde{\psi}_{j+1}^{k}\right\rangle \\
\chi_{N}^{k}=O \psi_{N}^{k},
\end{array}\right.
\end{aligned}
$$

where:

$$
\breve{\chi}_{j}^{\varepsilon}=\mathrm{e}^{-\frac{H_{0} \Delta T}{2 i}} \chi_{j}^{\varepsilon}, \widetilde{\psi}_{j}^{\varepsilon}=\mathrm{e}^{-\frac{H_{0} \Delta T}{2 i}} \psi_{j}^{\varepsilon}
$$

Subsequently, the initial value $\varepsilon^{0}$ of the monotonic schemes is considered fixed.

\subsection{Properties of sequence $\left(\varepsilon^{k}\right)_{k \in \mathbb{N}}$}

\subsubsection{Upper bound}

A first property of $\left(\varepsilon^{k}\right)_{k \in \mathbb{N}}$ and $\left(\tilde{\varepsilon}^{k}\right)_{k \in \mathbb{N}}$ defined in (17) and (18) is that these sequences are bounded. Indeed, the following result can be proved by induction (see [12]).

Lemma 3.1. Given an initial field $\varepsilon^{0}$, let us defined $M$ by:

$$
M=\max \left(\left\|\varepsilon^{0}\right\|_{\infty}, \max \left(1, \frac{\delta}{2-\delta}, \frac{\eta}{2-\eta}\right) \frac{\|O\|_{*}\|\mu\|_{*}}{\alpha}\right)
$$

The sequences $\left(\varepsilon^{k}\right)_{k \in \mathbb{N}}$ and $\left(\tilde{\varepsilon}^{k}\right)_{k \in \mathbb{N}}$ match the following conditions:

$$
\forall k \in \mathbb{N}, \forall j=0 \ldots N-1,\left|\varepsilon_{j}^{k}\right| \leq M,\left|\tilde{\varepsilon}_{j}^{k}\right| \leq M
$$

\subsubsection{Monotonic convergence}

A computation of the variation in the cost functional values leads to:

$$
\begin{aligned}
J_{\Delta T}\left(\varepsilon^{k+1}\right)-J_{\Delta T}\left(\varepsilon^{k}\right)= & \left\langle\psi_{N}^{k+1}-\psi_{N}^{k}|O| \psi_{N}^{k+1}-\psi_{N}^{k}\right\rangle \\
& +\sum_{j=0}^{N-1} 2 \operatorname{Re}\left\langle\breve{\chi}_{j+1}^{k}\left|\mathrm{e}^{\frac{\mu\left(\varepsilon_{j}^{k}-\tilde{\varepsilon}_{j}^{k}\right)}{i} \Delta T}-I d\right| \widetilde{\psi}_{j+1}^{k}\right\rangle-\alpha \Delta T\left(\left(\tilde{\varepsilon}_{j}^{k}\right)^{2}-\left(\varepsilon_{j}^{k}\right)^{2}\right) \\
& +\sum_{j=0}^{N-1} 2 \operatorname{Re}\left\langle\widetilde{\chi}_{j}^{k}\left|\mathrm{e}^{-\frac{\mu\left(\varepsilon_{j}^{k+1}-\tilde{\varepsilon}_{j}^{k}\right)}{i} \Delta T}-I d\right| \breve{\psi}_{j}^{k+1}\right\rangle-\alpha \Delta T\left(\left(\varepsilon_{j}^{k+1}\right)^{2}-\left(\tilde{\varepsilon}_{j}^{k}\right)^{2}\right) .
\end{aligned}
$$


Note that this equality holds for any sequence of controls $\left(\varepsilon^{k}\right)_{k \in \mathbb{N}}$ and $\left(\tilde{\varepsilon}^{k}\right)_{k \in \mathbb{N}}$. In the case of the implicit schemes (17)-(18), this expression reads:

$$
\begin{aligned}
J_{\Delta T}\left(\varepsilon^{k+1}\right)-J_{\Delta T}\left(\varepsilon^{k}\right)= & \left\langle\psi_{N}^{k+1}-\psi_{N}^{k}|O| \psi_{N}^{k+1}-\psi_{N}^{k}\right\rangle \\
& +\alpha \Delta T \sum_{j=0}^{N-1}\left(\frac{2}{\delta}-1\right)\left(\tilde{\varepsilon}_{j}^{k}-\varepsilon_{j}^{k+1}\right)^{2}+\left(\frac{2}{\eta}-1\right)\left(\varepsilon_{j}^{k}-\tilde{\varepsilon}_{j}^{k}\right)^{2} \\
= & \left\langle\psi_{N}^{k+1}-\psi_{N}^{k}|O| \psi_{N}^{k+1}-\psi_{N}^{k}\right\rangle \\
& +\alpha\left(\frac{2}{\delta}-1\right)\left\|\tilde{\varepsilon}^{k}-\varepsilon^{k+1}\right\|_{2}^{2}+\alpha\left(\frac{2}{\eta}-1\right)\left\|\varepsilon^{k}-\tilde{\varepsilon}^{k}\right\|_{2}^{2}
\end{aligned}
$$

This result, combined with the bounded character of $\left(\varepsilon^{k}\right)_{k \in \mathbb{N}}$ leads to the following theorem.

Theorem 3.2. The implicit schemes (17)-(18) ensure the monotonic convergence of the cost functional $J_{\Delta T}$ insofar as:

Furthermore there exists $l_{\varepsilon^{0}}$ such that:

$$
J_{\Delta T}\left(\varepsilon^{k}\right) \leq J_{\Delta T}\left(\varepsilon^{k+1}\right)
$$

$$
\lim _{k \rightarrow+\infty} J_{\Delta T}\left(\varepsilon^{k}\right)=l_{\varepsilon^{0}}
$$

Proof. Inequality (22) is a trivial consequence of (21), since $O$ is a positive operator. Moreover, we know that:

$$
\forall k \in \mathbb{N}, \quad J_{\Delta T}\left(\varepsilon^{k}\right) \leq\|O\|_{*},
$$

hence the existence of $l_{\varepsilon^{0}}$.

We keep the notation $l_{\varepsilon^{0}}$ in the sequel.

3.2.3. Limit points of $\left(\varepsilon^{k}\right)_{k \in \mathbb{N}}$

Having reached these preliminary results, we are now in a position to describe the limit points set $C_{\varepsilon^{0}}$ of sequence $\left(\varepsilon^{k}\right)_{k \in \mathbb{N}}$.

Lemma 3.3. Keeping the previous notations:

$$
C_{\varepsilon^{0}} \subset C,
$$

where $C$ is the set of critical points, defined by (12).

Proof. Consider a convergent subsequence $\left(\varepsilon^{k_{n}}\right)_{n \in \mathbb{N}}$ of $\left(\varepsilon^{k}\right)_{k \in \mathbb{N}}$ and its limit $\varepsilon^{\infty}$. By means of continuity and since $\left\|\varepsilon^{k_{n}}-\tilde{\varepsilon}^{k_{n}-1}\right\|_{2} \rightarrow 0$ (see (21)), we find the following limits:

$$
\begin{aligned}
\tilde{\varepsilon}^{k_{n}-1} & \rightarrow \varepsilon^{\infty}, \\
\mu^{*}\left(\varepsilon_{j}^{k}-\tilde{\varepsilon}_{j}^{k-1}\right) & \rightarrow \mu, \\
\psi^{k_{n}} & \rightarrow \psi^{\varepsilon^{\infty}}, \\
\chi^{k_{n}-1} & \rightarrow \chi^{\varepsilon^{\infty}} .
\end{aligned}
$$

When $n$ tends to $+\infty$, Equation (17) becomes:

$$
\forall j=0 \ldots N-1, \quad \varepsilon_{j}^{\infty}=-\frac{1}{\alpha} \operatorname{Im}\left\langle\widetilde{\chi}_{j}^{\infty}|\mu| \breve{\psi}_{j}^{\varepsilon^{\infty}}\right\rangle,
$$

which is the desired conclusion. 
Thanks to (19), a standard argument of compactness shows that:

$$
d\left(\varepsilon^{k}, C_{\varepsilon^{0}}\right) \rightarrow 0
$$

where $d\left(\varepsilon^{k}, C_{\varepsilon^{0}}\right)$ is the distance associated to $\|\cdot\|_{2}$ between $\varepsilon^{k}$ and set $C_{\varepsilon^{0}}$. Furthermore, inequality (22) then implies:

$$
J_{\Delta T}\left(C_{\varepsilon^{0}}\right)=l_{\varepsilon^{0}} .
$$

Finally, note that since $C$ is compact (see (12)), $C_{\varepsilon^{0}}$ is also compact.

\subsection{Estimates of the gradient}

This section introduces an estimate of the norm of the gradient at the points of the monotonic schemes. Let us recall that according to (11), we know that:

$$
\left\|\nabla J_{\Delta T}\left(\varepsilon^{k}\right)\right\|_{1}=2 \Delta T \sum_{j=0}^{N-1}\left|\operatorname{Im}\left\langle\widetilde{\chi}_{j}^{\varepsilon^{k}}|\mu| \breve{\psi}_{j}^{k}\right\rangle+\alpha \varepsilon_{j}^{k}\right| .
$$

Lemma 3.4. There exists $\lambda \geq 0$ such that:

$$
\left\|\nabla J_{\Delta T}\left(\varepsilon^{k}\right)\right\|_{1} \leq \lambda\left(\left\|\varepsilon^{k}-\tilde{\varepsilon}^{k-1}\right\|_{2}+\left\|\tilde{\varepsilon}^{k-1}-\varepsilon^{k-1}\right\|_{2}\right) .
$$

Proof. If we focus on one coefficient of $J_{\Delta T}\left(\varepsilon^{k}\right)$, we find:

$$
\begin{aligned}
\operatorname{Im}\left\langle\widetilde{\chi}_{j}^{\varepsilon^{k}}|\mu| \breve{\psi}_{j}^{k}\right\rangle+\alpha \varepsilon_{j}^{k}= & \operatorname{Im}\left\langle\widetilde{\chi}_{j}^{k-1}\left|\mu^{*}\left(\tilde{\varepsilon}_{j}^{k-1}-\varepsilon_{j}^{k}\right)\right| \breve{\psi}_{j}^{k}\right\rangle+\alpha \varepsilon_{j}^{k} \\
& +\operatorname{Im}\left\langle\widetilde{\chi}_{j}^{k-1}\left|\mu-\mu^{*}\left(\tilde{\varepsilon}_{j}^{k-1}-\varepsilon_{j}^{k}\right)\right| \breve{\psi}_{j}^{k}\right\rangle \\
& +\operatorname{Im}\left\langle\widetilde{\chi}_{j}^{\varepsilon^{k}}-\widetilde{\chi}_{j}^{k-1}|\mu| \breve{\psi}_{j}^{k}\right\rangle .
\end{aligned}
$$

Let us estimate each term of this decomposition. Definition (17) of the algorithm implies that:

$$
\operatorname{Im}\left\langle\widetilde{\chi}_{j}^{k-1}\left|\mu^{*}\left(\tilde{\varepsilon}_{j}^{k-1}-\varepsilon_{j}^{k}\right)\right| \breve{\psi}_{j}^{k}\right\rangle+\alpha \varepsilon_{j}^{k}=\frac{\alpha}{\delta}\left(\tilde{\varepsilon}_{j}^{k-1}-\varepsilon_{j}^{k}\right) .
$$

Then, thanks to (16), we obtain:

$$
\left|\operatorname{Im}\left\langle\widetilde{\chi}_{j}^{k}\left|\mu-\mu^{*}\left(\tilde{\varepsilon}_{j}^{k-1}-\varepsilon_{j}^{k}\right)\right| \breve{\psi}_{j}^{k}\right\rangle\right| \leq \Delta T\|\mu\|_{*}^{2}\|O\|_{*}\left|\varepsilon_{j}^{k}-\tilde{\varepsilon}_{j}^{k-1}\right| .
$$

Lastly, inequality (14) (see Rem. 2.2) yields:

$$
\left|\operatorname{Im}\left\langle\widetilde{\chi}_{j}^{\varepsilon^{k}}-\widetilde{\chi}_{j}^{k-1}|\mu| \breve{\psi}_{j}^{k}\right\rangle\right| \leq\|\mu\|_{*}^{2}\left(\left\|\varepsilon^{k}-\tilde{\varepsilon}^{k-1}\right\|_{1}+\|O\|_{*}\left\|\varepsilon^{k}-\varepsilon^{k-1}\right\|_{1}\right) .
$$

Combining (27)-(29) with (26), we come to equation (25) where

$$
\lambda=2 \sqrt{T}\left(\frac{\alpha}{\delta}+(T+\Delta T)\|\mu\|_{*}^{2}\left(1+\|O\|_{*}\right)\right) .
$$

\section{Convergence of the implicit SChemes}

The above mentioned results enable us to prove the convergence of the schemes. First we will present a general inequality due to Łojasiewicz $[8,9]$ (see also [3]), and use it to prove that $\left(\varepsilon^{k}\right)_{k \in \mathbb{N}}$ defined by $(17)-(18)$ is Cauchy. 


\section{1. Łojasiewicz inequality}

The Łojasiewicz inequality enables us to estimate the variation in an analytic function by its gradient. This result is detailed in the next theorem.

Theorem 4.1 (Łojasiewicz inequality). Let $\Gamma: \mathbb{R}^{n} \rightarrow \mathbb{R}$ be an analytic function in a neighborhood of a point a in $\mathbb{R}^{n}$. Then there exists $\sigma>0$ and $0<\theta \leq \frac{1}{2}$ such that:

$$
\forall x \in \mathbb{R}^{n}, x \in B(a, \sigma) \quad\|\nabla \Gamma(x)\| \geq|\Gamma(x)-\Gamma(a)|^{1-\theta},
$$

where $B(a, \sigma)$ denotes the ball centered in a with a radius equal to $\sigma,\|$.$\| is a norm on \mathbb{R}^{n}$.

The real number $\theta$ is called a Łojasiewicz exponent of $a$. A more precise result can be obtained if the Hessian matrix of $\Gamma$, denoted by $H_{\Gamma}(a)$, is invertible (see, e.g. [5]).

Lemma 4.2. Suppose that $H_{\Gamma}(a)$ is invertible, then there exists $\sigma>0$ and $\kappa>0$ such that:

$$
\forall x \in \mathbb{R}^{n}, x \in B(a, \sigma) \quad\|\nabla \Gamma(x)\| \geq \kappa|\Gamma(x)-\Gamma(a)|^{\frac{1}{2}} .
$$

In order to apply these results to our problem, let us define the shifted cost functional:

$$
\widetilde{J}_{\Delta T}(\varepsilon)=l_{\varepsilon^{0}}-J_{\Delta T}(\varepsilon)
$$

It is easy to check that $\widetilde{J}_{\Delta T}$ is analytic. Now, consider $a$ in $C_{\varepsilon^{0}}$. By means of $(24)$, we find that $\widetilde{J}_{\Delta T}(a)=0$. Consequently, Theorem 4.1 ensures that there exist $0<\theta_{a} \leq 1 / 2$ and $\sigma_{a}>0$ such that:

$$
\forall \varepsilon \in \mathbb{R}^{N},\|\varepsilon-a\|_{1}<\sigma_{a} \quad\left\|\nabla J_{\Delta T}(\varepsilon)\right\|_{1} \geq\left|\widetilde{J}_{\Delta T}(\varepsilon)\right|^{1-\theta_{a}} .
$$

The compactness of $C_{\varepsilon^{0}}$ enables us to extract from the set $\left\{B\left(a, \frac{\sigma_{a}}{2}\right), a \in C_{\varepsilon^{0}}\right\}$ a family $F_{\varepsilon^{0}}=\left\{B\left(a_{i}, \frac{\sigma_{a_{i}}}{2}\right)\right\}_{i \in I}$, where $I$ is a finite set of indexes such that:

$$
C_{\varepsilon^{0}} \subset F_{\varepsilon^{0}} .
$$

Let us denote by $\left.\left.\theta^{\prime} \in\right] 0,1 / 2\right]$, the lower bound of $\left\{\theta_{a_{i}}\right\}_{i \in I}$ and by $\sigma^{\prime}$, the lower bound of $\left\{\frac{\sigma_{a_{i}}}{2}\right\}_{i \in I}$. Given $\varepsilon$ such that $d\left(\varepsilon, C_{\varepsilon^{0}}\right)<\sigma^{\prime}$, there exists $i_{\varepsilon}$ such that $\varepsilon \in B\left(a_{i_{\varepsilon}}, \sigma_{a_{i_{\varepsilon}}}\right)$ and we come to the following version of Theorem 4.1:

Lemma 4.3. Keeping the above notations:

$$
\forall \varepsilon \in \mathbb{R}^{N}, d\left(\varepsilon, C_{\varepsilon^{0}}\right)<\sigma^{\prime}, \quad\left\|\nabla J_{\Delta T}(\varepsilon)\right\|_{1} \geq\left|\widetilde{J}_{\Delta T}(\varepsilon)\right|^{1-\theta^{\prime}} .
$$

In case $H_{J_{\Delta T}}$ is invertible on $C_{\varepsilon^{0}}$, a similar analysis can be led to obtain the corresponding version of Lemma 4.2 .

Lemma 4.4. Suppose that $H_{J_{\Delta T}}(\varepsilon)$ is invertible for all $\varepsilon \in C_{\varepsilon^{0}}$,

$$
\exists \sigma^{\prime \prime}>0, \exists \kappa^{\prime}>0, \forall \varepsilon \in \mathbb{R}^{N}, d\left(\varepsilon, C_{\varepsilon^{0}}\right)<\sigma^{\prime \prime}, \quad\left\|\nabla J_{\Delta T}(\varepsilon)\right\|_{1} \geq \kappa^{\prime}\left|\widetilde{J}_{\Delta T}(\varepsilon)\right|^{\frac{1}{2}} .
$$

Summarizing the above mentioned results, we have obtained that there exist $\widetilde{\sigma}>0, \widetilde{\kappa}>0$ and $\left.\widetilde{\theta} \in] 0, \frac{1}{2}\right]$ such that:

$$
d\left(\varepsilon, C_{\varepsilon^{0}}\right)<\widetilde{\sigma}, \quad\left\|\nabla J_{\Delta T}(\varepsilon)\right\|_{1} \geq \widetilde{\kappa}\left|\widetilde{J}_{\Delta T}(\varepsilon)\right|^{1-\widetilde{\theta}},
$$

with $\widetilde{\theta}=1 / 2$ when $H_{J_{\Delta T}}(\varepsilon)$ is invertible. 


\subsection{Cauchy property of the monotonic sequences}

It is now possible to prove the convergence of sequence $\left(\varepsilon^{k}\right)_{k \in \mathbb{N}}$.

Theorem 4.5. Sequence $\left(\varepsilon^{k}\right)_{k \in \mathbb{N}}$ defined by (17)-(18) converges towards a critical point of $J_{\Delta T}$.

Proof. First suppose that:

$$
\forall k \in \mathbb{N}, \widetilde{J}_{\Delta T}\left(\varepsilon^{k}\right) \neq 0 .
$$

According to (23), there exists $k_{0}$ such that (30) holds for all $\varepsilon^{k}$ with $k \geq k_{0}$, with $\tilde{\theta}=1 / 2$ if $H_{J_{\Delta T}}$ is invertible on $C_{\varepsilon^{0}}$. Consider an integer $k \geq k_{0}$. Thanks to the results above:

$$
\begin{aligned}
\left(\widetilde{J}_{\Delta T}\left(\varepsilon^{k}\right)\right)^{\widetilde{\theta}}-\left(\widetilde{J}_{\Delta T}\left(\varepsilon^{k+1}\right)\right)^{\widetilde{\theta}} & \geq \frac{\tilde{\theta}}{\left(\widetilde{J}_{\Delta T}\left(\varepsilon^{k+1}\right)\right)^{1-\tilde{\theta}}}\left(J_{\Delta T}\left(\varepsilon^{k+1}\right)-J_{\Delta T}\left(\varepsilon^{k}\right)\right) \\
& \geq \frac{\widetilde{\kappa} \tilde{\theta}}{\left\|\nabla J_{\Delta T}\left(\varepsilon^{k+1}\right)\right\|_{1}}\left(\left(\frac{2}{\delta}-1\right)\left\|\varepsilon^{k+1}-\tilde{\varepsilon}^{k}\right\|_{2}^{2}+\left(\frac{2}{\eta}-1\right)\left\|\tilde{\varepsilon}^{k}-\varepsilon^{k}\right\|_{2}^{2}\right) \\
& \geq \frac{\widetilde{\kappa} \tilde{\theta} a_{(\delta, \eta)}}{\left\|\nabla J_{\Delta T}\left(\varepsilon^{k+1}\right)\right\|_{1}}\left(\left\|\varepsilon^{k+1}-\tilde{\varepsilon}^{k}\right\|_{2}+\left\|\tilde{\varepsilon}^{k}-\varepsilon^{k}\right\|_{2}\right)^{2} \\
& \geq \frac{\widetilde{\kappa} \tilde{\theta} a_{(\delta, \eta)}}{\lambda}\left(\left\|\varepsilon^{k+1}-\tilde{\varepsilon}^{k}\right\|_{2}+\left\|\tilde{\varepsilon}^{k}-\varepsilon^{k}\right\|_{2}\right) \\
& \geq \frac{\widetilde{\kappa} \tilde{\theta} a_{(\delta, \eta)}}{\lambda}\left\|\varepsilon^{k+1}-\varepsilon^{k}\right\|_{2}
\end{aligned}
$$

where $a_{(\delta, \eta)}=\frac{1}{\max (\delta, \eta)}-\frac{1}{2}$. Given $q \in \mathbb{N}$, inequality (32) leads to:

$$
\begin{aligned}
\left(\widetilde{J}_{\Delta T}\left(\varepsilon^{k}\right)\right)^{\tilde{\theta}}-\left(\widetilde{J}_{\Delta T}\left(\varepsilon^{k+q}\right)\right)^{\tilde{\theta}} & \geq \frac{\widetilde{\kappa} \tilde{\theta} a_{(\delta, \eta)}}{\lambda} \sum_{l=k}^{k+q-1}\left\|\varepsilon^{l+1}-\varepsilon^{l}\right\|_{2} \\
& \geq \frac{\widetilde{\kappa} \widetilde{\theta} a_{(\delta, \eta)}}{\lambda}\left\|\varepsilon^{k+q}-\varepsilon^{k}\right\|_{2} .
\end{aligned}
$$

Since $\left(\left(\widetilde{J}_{\Delta T}\left(\varepsilon^{k}\right)\right)^{\widetilde{\theta}}\right)_{k \in \mathbb{N}}$ is a Cauchy sequence, we conclude that the sequence $\left(\varepsilon^{k}\right)_{k \in \mathbb{N}}$ is also Cauchy.

In case there exists $k_{1}$ such that $\widetilde{J}_{\Delta T}\left(\varepsilon^{k_{1}}\right)=0$, the monotonicity of the algorithm implies that $J_{\Delta T}\left(\varepsilon^{k_{1}}\right)=$ $J_{\Delta T}\left(\varepsilon^{k_{1}+1}\right)=J_{\Delta T}\left(\varepsilon^{k_{1}+2}\right)=\ldots$ and according to $(21)$ the sequence $\left(\varepsilon^{k}\right)_{k \in \mathbb{N}}$ is constant for $k \geq k_{1}$.

Lastly, the limit that has been reached belongs to $C$ according to Lemma 3.3.

Remark 4.6. A similar proof can be developed when $\delta=0, \eta \neq 0$ and $\delta \neq 0, \eta=0$. In case $\delta=0, \eta=0$, the convergence is trivial.

\section{RAte OF CONVERGENCE}

In this section, we will present both theoretical and numerical results regarding the rate of convergence of the discrete monotonic schemes.

\subsection{Theoretical results}

The rate of convergence can be evaluated by making a second use of Łojasiewicz inequality. The result is summarized in the next theorem. 
Theorem 5.1. Let us denote by $\varepsilon^{\infty}$ the limit of $\left(\varepsilon^{k}\right)_{k \in \mathbb{N}}$ defined by $(17)-(18)$ and by $\widetilde{\theta}$ and $\widetilde{\kappa}$ the real numbers appearing in $(30)$, where $C_{\varepsilon^{0}}=\left\{\varepsilon^{\infty}\right\}$.

If $\widetilde{\theta}<\frac{1}{2}$, then there exists $c>0$ such that:

$$
\left\|\varepsilon^{k}-\varepsilon^{\infty}\right\|_{2} \leq c k^{-\frac{\tilde{\theta}}{1-2 \theta}}
$$

If $\widetilde{\theta}=\frac{1}{2}$, then there exist $c^{\prime}$ and $\tau$ such that:

$$
\left\|\varepsilon^{k}-\varepsilon^{\infty}\right\|_{2} \leq c^{\prime} \mathrm{e}^{-\tau k}
$$

Proof. As in the proof of Theorem 4.5, let be $k_{0} \geq 0$ such that

$$
\forall l \geq k_{0} \quad\left\|\nabla J_{\Delta T}\left(\varepsilon^{l}\right)\right\|_{1} \geq \widetilde{\kappa}\left|\widetilde{J}_{\Delta T}\left(\varepsilon^{l}\right)\right|^{1-\widetilde{\theta}} .
$$

Let us fix $k \geq k_{0}$ and introduce $\Delta^{k}$ defined by:

$$
\Delta^{k}=\sum_{l=k}^{\infty}\left\|\varepsilon^{l+1}-\tilde{\varepsilon}^{l}\right\|_{2}+\left\|\tilde{\varepsilon}^{l}-\varepsilon^{l}\right\|_{2} .
$$

Sticking to a general way of reasoning, we may assume that $\Delta^{k}>0$ for any $k \geq k_{0}$. By summing (31) between $k$ and $+\infty$, we obtain:

$$
\left(\widetilde{J}_{\Delta T}\left(\varepsilon^{k}\right)\right)^{\tilde{\theta}} \geq \frac{\widetilde{\kappa} \tilde{\theta} a_{(\delta, \eta)}}{\lambda} \Delta^{k} .
$$

This estimate, combined with (35), yields:

$$
\left\|\nabla J_{\Delta T}\left(\varepsilon^{k}\right)\right\|_{1} \geq \widetilde{\kappa}\left(\frac{\widetilde{\kappa} \widetilde{\theta} a_{(\delta, \eta)}}{\lambda} \Delta^{k}\right)^{\frac{1-\tilde{\theta}}{\tilde{\theta}}} .
$$

From Lemma 3.4, we get:

$$
\lambda\left(\Delta^{k-1}-\Delta^{k}\right) \geq \widetilde{\kappa}\left(\frac{\widetilde{\kappa} \widetilde{\theta} a_{(\delta, \eta)}}{\lambda} \Delta^{k}\right)^{\frac{1-\tilde{\theta}}{\tilde{\theta}}}
$$

which can be rewritten as follows:

$$
\frac{\Delta^{k-1}-\Delta^{k}}{\left(\Delta^{k}\right)^{\beta}} \geq \nu
$$

with $\nu>0$ and $\beta=\frac{1-\tilde{\theta}}{\tilde{\theta}}$.

Suppose that $\widetilde{\theta}=\frac{1}{2}$, i.e., $\beta=1$. Equation (36) reads:

$$
(1+\nu)^{k_{0}} \Delta^{k_{0}}\left(\frac{1}{1+\nu}\right)^{k} \geq \Delta^{k}
$$

and (34) is proved with $c^{\prime}=(1+\nu)^{k_{0}} \Delta^{k_{0}}$ and $\tau=\ln (1+\nu)$.

Now let us consider the case in which $\widetilde{\theta}<\frac{1}{2}$. Let $\left.r \in\right] 0,1[$, and first suppose that:

$$
\left(\Delta^{k}\right)^{\beta} \geq r\left(\Delta^{k-1}\right)^{\beta}
$$


Since $1-\beta<0$, the function $s \mapsto s^{1-\beta}$ is convex and consequently:

$$
\begin{aligned}
\left(\Delta^{k}\right)^{1-\beta}-\left(\Delta^{k-1}\right)^{1-\beta} & \geq(\beta-1) \frac{\Delta^{k-1}-\Delta^{k}}{\left(\Delta^{k-1}\right)^{\beta}} \\
& \geq(\beta-1) r \frac{\Delta^{k-1}-\Delta^{k}}{\left(\Delta^{k}\right)^{\beta}} \\
& \geq(\beta-1) r \nu
\end{aligned}
$$

In the other case:

$$
\left(\Delta^{k}\right)^{\beta} \leq r\left(\Delta^{k-1}\right)^{\beta}
$$

and consequently:

$$
\left(\Delta^{k}\right)^{1-\beta}-\left(\Delta^{k-1}\right)^{1-\beta} \geq\left(\Delta^{k}\right)^{1-\beta}-\left(r^{\frac{1}{\beta}} \Delta^{k}\right)^{1-\beta}=\left(1-r^{\frac{1-\beta}{\beta}}\right)\left(\Delta^{k}\right)^{1-\beta} \geq\left(1-r^{\frac{1-\beta}{\beta}}\right)\left(\Delta^{k_{0}}\right)^{1-\beta} .
$$

Thus, in any case, there exists $\nu^{\prime} \geq 0$ such that:

$$
\left(\Delta^{k}\right)^{1-\beta}-\left(\Delta^{k-1}\right)^{1-\beta} \geq \nu^{\prime} .
$$

Consider now $k^{\prime}>k$. Inequality (37) implies that for small enough a $c$, we get:

$$
\Delta^{k^{\prime}} \leq\left(\nu^{\prime}\left(k^{\prime}-k\right)+\left(\Delta^{k}\right)^{2-\frac{1}{\theta}}\right)^{-\frac{\tilde{\theta}}{1-2 \theta}} \leq c k^{\prime-\frac{\tilde{\theta}}{1-2 \theta}}
$$

and (33) follows.

Remark 5.2. Thanks to Lemma 2.1, we have thus obtained that if:

$$
\alpha>2\|\mu\|_{*}^{2}\|O\|_{*} T
$$

the convergence of the schemes is at least linear.

\subsection{Numerical results}

In order to test the performance of the algorithm we have chosen a case already treated in the literature. The system under consideration is the $O-H$ bond that vibrates in a Morse type potential. We refer to [20] for the numerical details concerning this system. The objective is to localize the wavefunction at a given location $x^{\prime}$; this is expressed through the requirement that $\langle\psi(T)|O| \psi(T)\rangle$ is maximized, where the observable $O$ is defined by $O(x)=\frac{\gamma_{0}}{\sqrt{\pi}} \mathrm{e}^{-\gamma_{0}^{2}\left(x-x^{\prime}\right)^{2}}$. In our test, $\beta=1,\|\mu\|_{*}=0.68151,\|O\|_{*}=12.375$ and $T=131000$. Numerical tests carried out on this example show that though (38) is not true at all, the convergence of the algorithm is linear, as it appears in Figure 1. Therefore some further analysis has to be done to improve Lemma 2.1 and the results of Section 5.1.

\section{APPENDIX: CONVERGENCE OF THE EXPLICIT DISCRETE MONOTONIC SCHEME}

In [12], an explicit scheme has also been presented. The proof of convergence of this scheme, although analogous to that of the implicit scheme, is slightly more elaborate. We briefly introduce here a sufficient condition for convergence. 


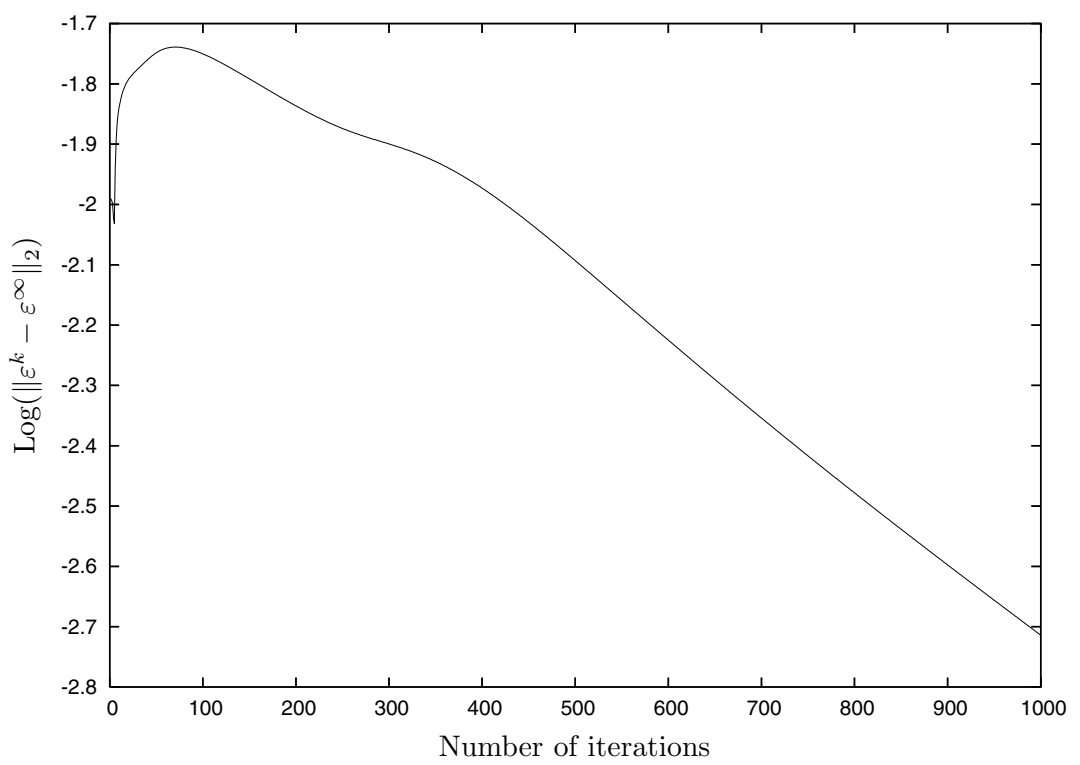

FiguRE 1. The rate of convergence is linear after the 500-th iteration.

\section{Definition of the scheme}

Given the initial control amplitudes $\varepsilon^{0}, \tilde{\varepsilon}^{0}$ and their associated state $\psi^{0}$ and adjoint state $\chi^{0}$, suppose that for some $k \geq 1, \psi^{k-1}, \chi^{k-1}, \varepsilon^{k-1}, \tilde{\varepsilon}^{k-1}$ have already been computed. The iterated quantities of $\psi^{k}, \chi^{k}, \varepsilon^{k}, \tilde{\varepsilon}^{k}$ are defined as follows:

$$
\begin{aligned}
& \left\{\begin{array}{l}
\psi_{j+1}^{k}=\mathrm{e}^{\frac{H_{0} \Delta T}{2 i}} \mathrm{e}^{\frac{V-\mu \varepsilon_{j}^{k}}{i} \Delta T} \mathrm{e}^{\frac{H_{0} \Delta T}{2 i}} \psi_{j}^{k} \\
\varepsilon_{j}^{k}=\left(1-\delta_{j}^{k}\right) \tilde{\varepsilon}_{j}^{k-1}-\frac{\delta_{j}^{k}}{\alpha} \operatorname{Im}\left\langle\widetilde{\chi}_{j}^{k-1}|\mu| \breve{\psi}_{j}^{k}\right\rangle \\
\psi_{0}^{k}=\psi_{i n i t},
\end{array}\right. \\
& \left\{\begin{array}{l}
\chi_{j}^{k}=\mathrm{e}^{-\frac{H_{0} \Delta T}{2 i}} \mathrm{e}^{\frac{-V+\mu \tilde{\varepsilon}_{j}^{k}}{i} \Delta T} \mathrm{e}^{-\frac{H_{0} \Delta T}{2 i}} \chi_{j+1}^{k} \\
\tilde{\varepsilon}_{j}^{k}=\left(1-\eta_{j}^{k}\right) \varepsilon_{j}^{k}-\frac{\eta_{j}^{k}}{\alpha} \operatorname{Im}\left\langle\breve{\chi}_{j+1}^{k}|\mu| \widetilde{\psi}_{j+1}^{k}\right\rangle \\
\chi_{N}^{k}=O \psi_{N}^{k},
\end{array}\right.
\end{aligned}
$$

where:

$$
\delta_{j}^{k}=\frac{\alpha}{\alpha+\Delta T \operatorname{Re}\left\langle\widetilde{\chi}_{j}^{k-1}\left|\mu^{2}\right| \breve{\psi}_{j}^{k}\right\rangle},
$$

and:

$$
\eta_{j}^{k}=\frac{\alpha}{\alpha+\Delta T \operatorname{Re}\left\langle\breve{\chi}_{j+1}^{k}\left|\mu^{2}\right| \widetilde{\psi}_{j+1}^{k}\right\rangle} .
$$




\section{Properties of sequence $\left(\varepsilon^{k}\right)_{k \in \mathbb{N}}$}

\subsection{Upper bound}

The first step of the convergence analysis is to obtain an upper bound for the fields. Suppose that:

$$
\alpha>2 \Delta T\|\mu\|_{*}^{2}\|O\|_{*}
$$

This condition ensures that:

$$
\forall k \in \mathbb{N}, \forall j=0 \ldots N-1, \delta_{j}^{k+1} \in\left[0,2 \frac{\alpha}{\alpha+e}\right], \eta_{j}^{k+1} \in\left[0,2 \frac{\alpha}{\alpha+e}\right]
$$

where $e=\alpha-2 \Delta T\|\mu\|_{*}^{2}\|O\|_{*}$. An analysis similar to that in the proof of Lemma 3.1 (see [12]) gives the following result:

Lemma 5.3. Suppose that (41) is fulfilled. Given an initial field $\varepsilon^{0}$, let us define $M$ by:

$$
M=\max \left(\left\|\varepsilon^{0}\right\|_{\infty}, \max \left(1, \frac{1}{e}\right)\|O\|_{*}\|\mu\|_{*}\right)
$$

The sequences $\left(\varepsilon^{k}\right)_{k \in \mathbb{N}}$ and $\left(\tilde{\varepsilon}^{k}\right)_{k \in \mathbb{N}}$ meet the requirements:

$$
\forall k \in \mathbb{N}, \forall j=0 \ldots N-1,\left|\varepsilon_{j}^{k}\right| \leq M,\left|\tilde{\varepsilon}_{j}^{k}\right| \leq M
$$

\subsection{Monotonic convergence}

In the case of the explicit scheme, equation (20) cannot be explicitly simplified as was the case for the implicit schemes. However, the Taylor-Lagrange formula stipulates the existence of two real numbers $x_{j}^{k}$ and $y_{j}^{k}$ in $[-M, M]$ such that:

$$
\begin{aligned}
\operatorname{Re}\left\langle\breve{\chi}_{j+1}^{k}\left|\mathrm{e}^{\frac{\mu\left(\varepsilon_{j}^{k}-\tilde{\varepsilon}_{j}^{k}\right)}{i} \Delta T}-I d\right| \widetilde{\psi}_{j+1}^{k}\right\rangle= & \operatorname{Im}\left\langle\breve{\chi}_{j+1}^{k}|\mu| \widetilde{\psi}_{j+1}^{k}\right\rangle\left(\varepsilon_{j}^{k}-\tilde{\varepsilon}_{j}^{k}\right) \Delta T \\
& -\operatorname{Re}\left\langle\breve{\chi}_{j+1}^{k}\left|\mu^{2} \mathrm{e}^{-i \mu x_{j}^{k} \Delta T}\right| \widetilde{\psi}_{j+1}^{k}\right\rangle \frac{\left(\varepsilon_{j}^{k}-\tilde{\varepsilon}_{j}^{k}\right)^{2} \Delta T^{2}}{2},
\end{aligned}
$$

and:

$$
\begin{aligned}
\operatorname{Re}\left\langle\widetilde{\chi}_{j}^{k}\left|\mathrm{e}^{i \mu\left(\varepsilon_{j}^{k+1}-\tilde{\varepsilon}_{j}^{k}\right) \Delta T}-I d\right| \breve{\psi}_{j}^{k+1}\right\rangle= & -\operatorname{Im}\left\langle\widetilde{\chi}_{j}^{k}|\mu| \breve{\psi}_{j}^{k+1}\right\rangle\left(\varepsilon_{j}^{k+1}-\tilde{\varepsilon}_{j}^{k}\right) \Delta T \\
& +\operatorname{Re}\left\langle\widetilde{\chi}_{j}^{k}\left|\mu^{2} \mathrm{e}^{i \mu y_{j}^{k} \Delta T}\right| \breve{\psi}_{j}^{k+1}\right\rangle \frac{\left(\varepsilon_{j}^{k+1}-\tilde{\varepsilon}_{j}^{k}\right)^{2} \Delta T^{2}}{2}
\end{aligned}
$$


Combining (42) and (43) with the definitions of $\varepsilon_{j}^{k}$ and $\tilde{\varepsilon}_{j}^{k}$ given by (39)-(40), we can conclude that (20) can be written as follows:

$$
\begin{aligned}
J_{\Delta T}\left(\varepsilon^{k+1}\right)-J_{\Delta T}\left(\varepsilon^{k}\right)= & \left\langle\psi_{N}^{k+1}-\psi_{N}^{k}|O| \psi_{N}^{k+1}-\psi_{N}^{k}\right\rangle \\
& +\Delta T \sum_{j=0}^{N-1}\left(\varepsilon_{j}^{k}-\tilde{\varepsilon}_{j}^{k}\right)^{2}[\alpha \\
& +\Delta T \operatorname{Re}\left\langle\breve{\chi}_{j+1}^{k}\left|\mu^{2}\right| \widetilde{\psi}_{j+1}^{k}\right\rangle \\
& \left.+\Delta T \operatorname{Re}\left\langle\breve{\chi}_{j+1}^{k}\left|\mu^{2}\left(I d-\mathrm{e}^{-i \mu x_{j}^{k} \Delta T}\right)\right| \widetilde{\psi}_{j+1}^{k}\right\rangle\right] \\
& +\Delta T \sum_{j=0}^{N-1}\left(\tilde{\varepsilon}_{j}^{k}-\varepsilon_{j}^{k+1}\right)^{2}\left[\alpha+\Delta T \operatorname{Re}\left\langle\widetilde{\chi}_{j}^{k}\left|\mu^{2}\right| \breve{\psi}_{j}^{k+1}\right\rangle\right. \\
& \left.+\Delta T \operatorname{Re}\left\langle\widetilde{\chi}_{j}^{k}\left|\mu^{2}\left(I d-\mathrm{e}^{i \mu y_{j}^{k} \Delta T}\right)\right| \breve{\psi}_{j}^{k+1}\right\rangle\right] .
\end{aligned}
$$

We are now in a position to claim the following result, which is the equivalent of formula (21) associated with the implicit schemes.

Lemma 5.4. Suppose that the real number $\gamma=\alpha-3 \Delta T\|\mu\|_{*}^{2}\|O\|_{*}$ is strictly positive. Then the explicit scheme is monotonic and:

$$
J_{\Delta T}\left(\varepsilon^{k+1}\right)-J_{\Delta T}\left(\varepsilon^{k}\right) \geq \gamma\left[\left\|\varepsilon_{j}^{k}-\tilde{\varepsilon}_{j}^{k}\right\|_{2}^{2}+\left\|\tilde{\varepsilon}_{j}^{k}-\varepsilon_{j}^{k+1}\right\|_{2}^{2}\right] .
$$

\section{Convergence}

The end of the proof of convergence of the explicit scheme can then be done in a similar way as for implicit schemes (in particular, the proof of Lem. 3.4 can be easily adapted). The next theorem summarizes the result reached.

Theorem 5.5. Suppose that:

$$
\alpha>3 \Delta T\|\mu\|_{*}^{2}\|O\|_{*}
$$

Then the sequence $\left(\varepsilon^{k}\right)_{k \in \mathbb{N}}$ defined by (39)-(40) converges towards a critical point of $J_{\Delta T}$.

Acknowledgements. We are very grateful to J. Bolte (Laboratoire de Combinatoire et Optimisation, Université P. \& M. Curie, Paris), Y. Maday (Laboratoire Jacques-Louis Lions, Université P. \& M. Curie, Paris) and G. Turinici (CEREMADE, Université Paris Dauphine) for the helpful discussions that we have had on this topic.

\section{REFERENCES}

[1] A.D. Bandrauk and H. Shen, Exponential split operator methods for solving coupled time-dependent Schrödinger equations. J. Chem. Phys. 99 (1993) 1185-1193.

[2] K. Beauchard, Local controllability of a 1D Schrödinger equation. J. Math. Pures Appl. 84 (2005) 851-956.

[3] J. Bolte and H. Attouch, On the convergence of the proximal point algorithm for nonsmooth functions involving analytic features. Math. Program. (to appear).

[4] E. Brown and H. Rabitz, Some mathematical and algorithmic challenges in the control of quantum dynamics phenomena. $J$. Math. Chem. 31 (2002) 17-63.

[5] A. Haraux, M.A. Jendoubi and O. Kavian, Rate of decay to equilibrium in some semilinear parabolic equations. J. Evol. Equ. 3 (2003) 463-484.

[6] K. Ito and K. Kunisch, Optimal bilinear control of an abstract Schrödinger equation. SIAM J. Cont. Opt. (to appear).

[7] R. Judson and H. Rabitz, Teaching lasers to control molecules. Phys. Rev. Lett 6810 (1992) 1500-1503.

[8] S. Łojasiewicz, Une propriété topologique des sous-ensembles analytiques réels. Colloques internationaux du CNRS, Les équations aux dérivées partielles 117 (1963).

[9] S. Łojasiewicz, Sur la géométrie semi- et sous-analytique. Ann. Inst. Fourier 43 (1993) 1575-1595.

[10] Y. Maday and G. Turinici, New formulations of monotonically convergent quantum control algorithms. J. Chem. Phys 118 18 (2003) 8191-8196.

[11] Y. Maday, J. Salomon and G. Turinici, Discretely monotonically convergent algorithm in quantum control, in Proc. LHMNLC03 IFAC conference, Sevilla (2003) 321-324. 
[12] Y. Maday, J. Salomon and G. Turinici, Monotonic time-discretized schemes in quantum control. Num. Math. 103 (2006) $323-338$.

[13] H. Rabitz, G. Turinici and E. Brown, Control of quantum dynamics: Concepts, procedures and future prospects, in Computational Chemistry, Special Volume (C. Le Bris Editor) of Handbook of Numerical Analysis, Vol. X, edited by Ph.G. Ciarlet, Elsevier Science B.V. (2003).

[14] J. Salomon, Limit points of the monotonic schemes in quantum control, in Proc. 44th IEEE Conference on Decision and Control, Sevilla (2005).

[15] S. Shi, A. Woody and H. Rabitz, Optimal control of selective vibrational excitation in harmonic linear chain molecules. $J$. Chem. Phys. 88 (1988) 6870-6883.

[16] G. Strang, Accurate partial difference methods. I: Linear cauchy problems. Arch. Rat. Mech. An. 12 (1963) 392-402.

[17] J. Szeftel, Absorbing boundary conditions for nonlinear Schrödinger equation. Num. Math. 104 (2006) 103-127.

[18] D. Tannor, V. Kazakov and V. Orlov, Control of photochemical branching: Novel procedures for finding optimal pulses and global upper bounds, in Time Dependent Quantum Molecular Dynamics, J. Broeckhove, L. Lathouwers Eds., Plenum (1992) $347-360$.

[19] T.N. Truong, J.J. Tanner, P. Bala, J.A. McCammon, D.J. Kouri, B. Lesyng and D.K. Hoffman, A comparative study of time dependent quantum mechanical wave packet evolution methods. J. Chem. Phys. 96 (1992) 2077-2084.

[20] W. Zhu and H. Rabitz, A rapid monotonically convergent iteration algorithm for quantum optimal control over the expectation value of a positive definite operator. J. Chem. Phys. 109 (1998) 385-391. 